POS $\quad$ PROCEEDINGS

\title{
Sterile Neutrino Search at the NEOS Experiment
}

\section{Youngju Ko*, Chang-Hwan Jang, Kim Siyeon}

Department of Physics, Chung-Ang University, Seoul 06974, Korea

E-mail: godpapa7egmail.com

\section{Kyung-Kwang Joo, Ba-Ro Kim}

Department of Physics, Chonnam National University, Gwangju 61186, Korea

Eun-Ju Jeon, Young-Duk Kim, Jaison Lee, Moo-Hyun Lee, Yoo-Min Oh, Hyang-Kyu Park, Kang-Soon Park

Center for Underground Physics, Institute for Basic Science, Daejeon 34047, Korea

\section{Bo-Young Han, Gwang-Min Sun}

Neutron Science Division, Korea Atomic Energy Research Institute, Daejeon 34057, Korea

\section{Hong-Joo Kim, Joo-Young Lee}

Department of Physics, Kyungpook National University, Daegu 41566, Korea

\section{Hyunsoo Kim, Jinyu Kim, Kyung-Min Seo}

Department of Physics and Astronomy, Sejong University, Seoul 05006, Korea

\begin{abstract}
In order to test the existence of the sterile neutrino, NEOS experiment was performed from August 2015 to May 2016. The prompt energy spectrum from the inverse beta decay of electron antineutrino from a $2.8 \mathrm{GW}_{\text {th }}$ reactor was measured at $24 \mathrm{~m}$ distance. The number of measured IBD candidates is about 2000 per day during reactor-on period and a ratio of signal to background is about 22. The result shows no strong evidence of active-to-sterile neutrino. An exclusion-limit curve for $3+1$ hypothesis is found at $90 \%$ CL. via shape-only analysis.
\end{abstract}

The 19th International Workshop on Neutrinos from Accelerators-NUFACT2017

25-30 September, 2017

Uppsala University, Uppsala, Sweden

\footnotetext{
* Speaker.
} 


\section{Introduction}

LSND [U] and MiniBooNE []] observed an evidence for $\bar{v}_{\mu} \rightarrow \bar{v}_{e}$ oscillation, which is excess in the number of measured electron antineutrino. Meanwhile, GALLEX [B]][䧃] and SAGE [可] [可] measured $\bar{v}_{e} \rightarrow \bar{v}_{e}$ disappearance for calibration, and they found deficits in the number of survived neutrinos. These can be explained by the $3+1 v$ framework, where three active neutrinos and one sterile neutrino are assumed. Thereafter, Reactor Antineutrino Anomaly [ర]] (RAA) including the reanalysis of the short-baseline reactor neutrino experiments also claims the deficit of $\bar{v}_{e} \rightarrow \bar{v}_{e}$ disappearance. Those results bring up the possibility of the existence of sterile neutrinos in $3+1 v$ framework, and more accurate short baseline reactor neutrino experiments has been demanded. Neutrino Experiment for Oscillation at Shrot baseline (NEOS) is a reactor neutrino experiment at short baseline to search for sterile neutrino in the $3+1 v$ framework.

\section{Experimental Site and Detector}

The experimental site is Hanbit Nuclear Power Plant (HNPP) in Younggwang, Korea. The HNPP has six reactor units, and the detector is located at the fifth unit. The reactor core has the thermal power of $2.8 \mathrm{GW}$, and the active core has cylindrical shape of which the diameter is $3.1 \mathrm{~m}$ and the height is $3.8 \mathrm{~m}$. The low enriched uranium fuel is used for reactor operation and one third of 177 fuel rods are replaced after each burn up cycle. The detector is located in the tendon gallery of the containment building, where at least 20 meter water equivalent overburden is provided. The baseline between the active core center and the detector is $23.7 \mathrm{~m}$, giving the most sensitive range for $\mathrm{eV}$ scale sterile neutrino as shown in left figure of Figs.Ш.

The NEOS detector consists of an active target with 8-inch photomultiplier tubes (PMTs), some passive shieldings, and muon veto counter as shown in right figure of Figs.W. The target is homogeneous liquid scintillator (LS) in a cylindrical tank that has about $1000 \mathrm{~L}$ inner volume. The $0.5 \%$ Gd is loaded in the LS. The LS has been developed for the improvement of pulse shape discrimination (PSD), mixing LAB- and DIN-based LS with a ratio of 9:1. The 38 PMTs (Hamamatsu R5912) are used to detect the signals from the active target. They are installed in two buffer tanks
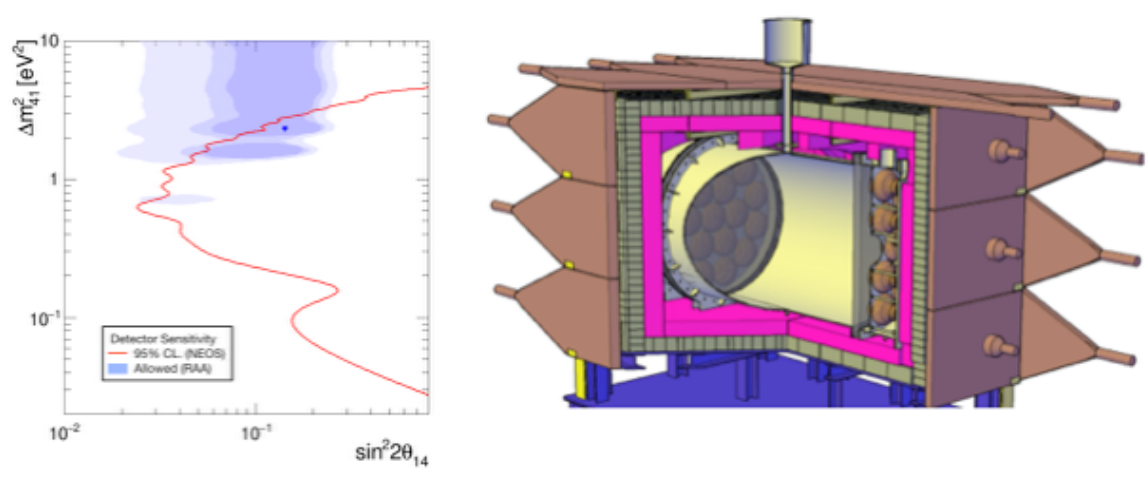

Figure 1: (Left) Sensitivity of NEOS detector at 95\% CL. (red line). The blue areas are allowed region by RAA. (Right) CAD drawing of NEOS detector. 
filled with mineral oil at both sides of the target tank, and there are two acrylic windows between the target tank and the buffer tanks.

We use two layers of passive shieldings. The inner layer is borated polyethylene (B-PE) of $10-\mathrm{cm}$ thickness against external neutrons, and the outer layer is $10-\mathrm{cm}$ thick lead against external $\gamma \mathrm{s}$. This ordering is a good way to shield neutrons produced by cosmic rays at lead layers. The outermost layer is active shielding with several muon detectors for muon veto, which consists of 155 -cm thick plastic scintillator panels with 3- or 5-inch PMTs. The muon detectors cover all directions except the bottom side. The DAQ system has $500 \mathrm{MS} / \mathrm{s}$ flash analog-to-digital converters (FADC) to record waveforms for PSD, 62.5 MS/s ADC for muon detectors, and trigger control board (TCB). The detector was installed in July 2015, and the physical data is taken from October in 2015 to May in 2016. The period includes not only reactor-on data but also -off data, and the DAQ efficiency is about $90 \%$.

The energy calibration is performed with several sources in two kinds. One refers to the pointlike radioactive sources such as ${ }^{137} \mathrm{Cs},{ }^{60} \mathrm{Co},{ }^{252} \mathrm{Cf}$, and $\mathrm{PoBe}$, which are used once every week. And there are internal and external radioactivies which are continuous and volume sources. The ${ }^{40} \mathrm{~K}$ in PMT glass emits $1.46-\mathrm{MeV} \gamma$-rays and the ${ }^{208} \mathrm{Tl}$ in B-PE also emits $2.61-\mathrm{MeV} \gamma$-rays which is used for correction of charge drift in time mainly due to temperature variation. There are $\alpha$ and $\beta$ events from the natural radon contamination in the LS. The $\alpha$ and $\beta$ events are used for correction of position dependence due to geometrical effect and validation of the Monte Carlo (MC) simulation, respectively. The 3D calibration is also performed with ${ }^{137} \mathrm{Cs},{ }^{60} \mathrm{Co}$, and ${ }^{252} \mathrm{Cf}$ sources at various positions inside the active target volume. They are used for $\mathrm{MC}$ tuning about position dependence and $\gamma$ 's escaping from target due to detector geometry including optical properties and the small size of the detector, respectively.
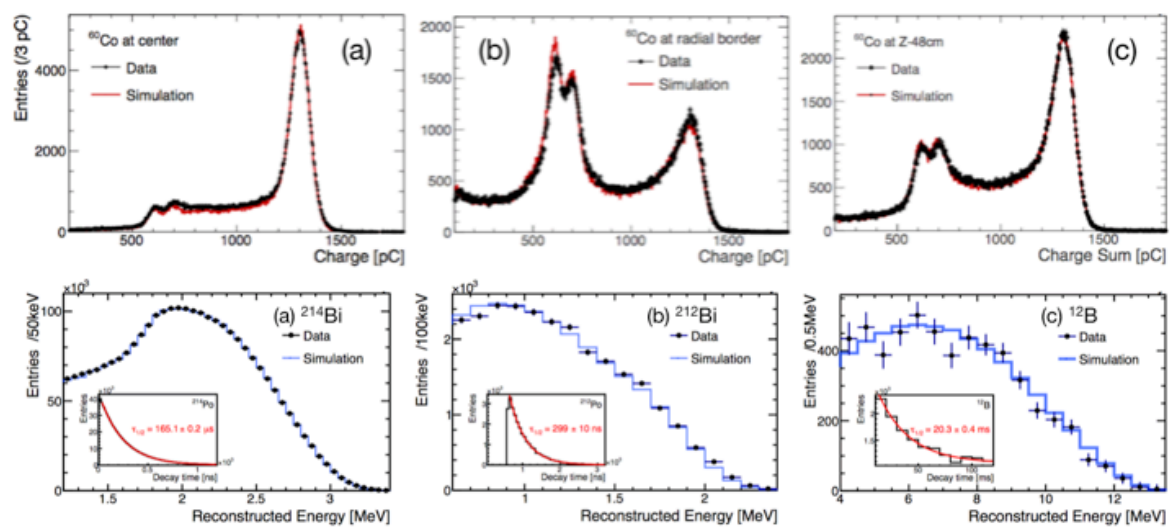

Figure 2: (Top) Charge distribution of 3D calibration with ${ }^{60} \mathrm{Co}$ at center (a), radial border (b), and near the PMTs (c). The black dots are data and red lines are MC. (Bottom) Beta spectra of ${ }^{214} \mathrm{Bi}(\mathrm{a}),{ }^{212} \mathrm{Bi}(\mathrm{b})$, and ${ }^{12} \mathrm{~B}$ (c). The black dots are data and blue lines are MC, and inserted small plots show the decay time with fitted exponential function. 

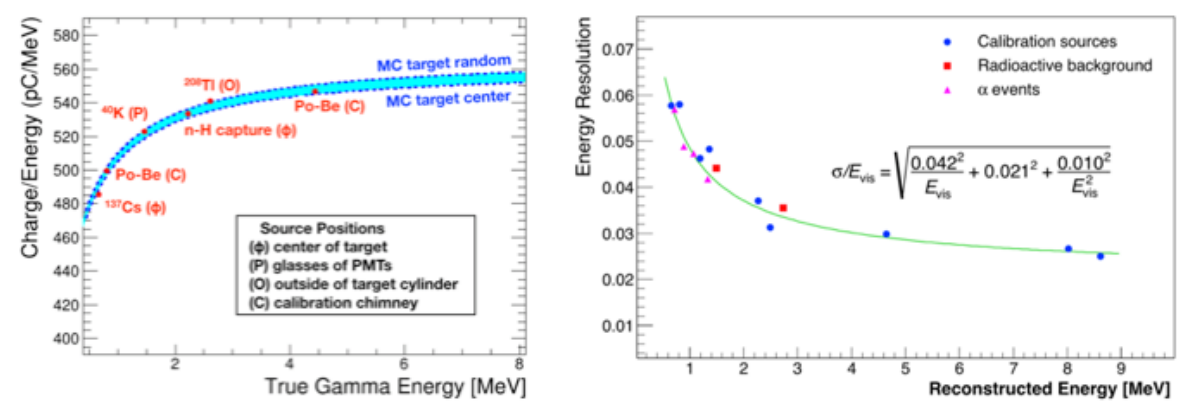

Figure 3: (Left) The non-linearity between charge and energy. The charge is converted to energy with this function. (Right) Energy resolution of the NEOS detector for full peak.

\section{Reconstruction}

A Geant 4 based MC simulation is developed to reproduce the detector response, by tuning the optical properties of LS, PMT parameters. The simulation includes physical process from the trigger level at the electronics. The simulation successfully reproduces the detector response to the calibration sources as shown in upper figures of Figs.】. There are non-linearity between charge and true energy due to quenching and Cherenkov effect. For that, only single $\gamma$ sources are used for conversion from charge to energy, and the left figure of Figs. [3 shows the non-linearity of data with MC. And then, the MC is verified with $\beta$ spectra as shown in lower figures of Figs.】. They are distributed throughout the LS, and the MC and data are in good agreement.

The energy of events are reconstructed with a non-linearity function, and the events that have energies above $0.6 \mathrm{MeV}$ are used for the analysis. With the single events of various sources, the energy resolution of full peak is around $4.8 \%$ at $1 \mathrm{MeV}$ as shown in right figure of Figs.[3. The IBD events are selected by paring of prompt and delayed signals. The delayed signal should have energy in 4-10 MeV range, with the timing coincidence within 1-30 $\mu$ s after a prompt signal.

There are some additional criteria to minimize the background. First, events within $150 \mu \mathrm{s}$
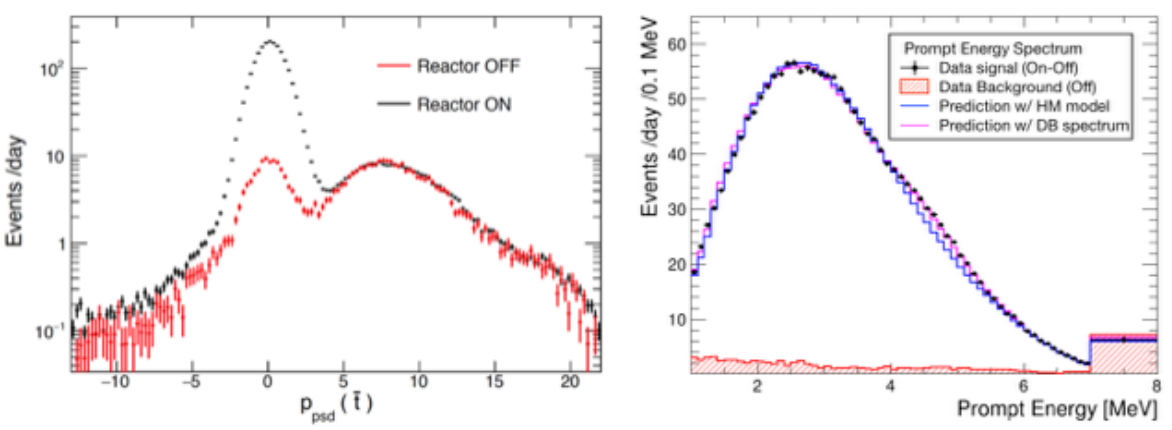

Figure 4: (Left) PSD distribution of IBD candidates. The black dots are reactor-on data and the red dots are reactor-off data. (Right) Prompt energy spectrum with MC. The black dots are data signals and the red area is background data in reactor-off period. The blue and magenta lines are produced by MC with two models. 

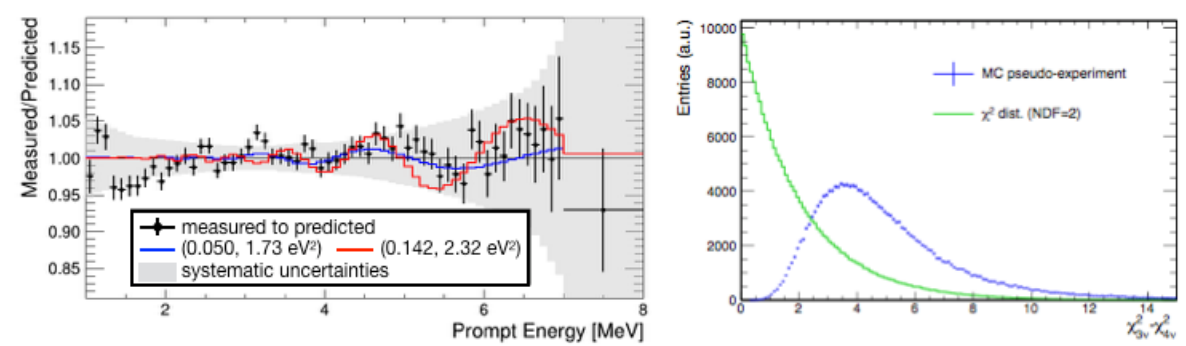

Figure 5: (Left) Measured to predicted ratio with uncertainties (black dots). The gray area is total systematic errors, the blue and red line shows the best fits of NEOS and RAA, respectively. (Right) $\Delta \chi^{2}$ distribution via pseudo-experiments (blue dots). The distribution shows that the $p$-value is $22 \%$ with $\Delta \chi^{2}=6.5$. The green line is $c h i^{2}$ distribution with $2 \mathrm{NDF}$.

after a muon counter event are vetoed. Another is the multiplicity cut that there should be no other event than the prompt-delayed pair within $30 \mu \mathrm{s}$ before or $150 \mu \mathrm{s}$ after the prompt event. The multiplicity cut is for reducing background due to multiple neutrons. Finally, the PSD cut is applied for reducing of background caused by fast neutrons. More than $70 \%$ of the rest of the background events are rejected using PSD, as shown in Figs.⿴囗十 left. After applying all the criteria described so far, prompt energy spectrum is obtained as shown in right figure of Figs.G. In the figure, there are spectra by MC with two models, one is HM model and the other is the absolute spectrum measured by Daya Bay experiment [12] which is used for oscillation analysis.

\section{Oscillation Analysis}

The $\chi^{2}$ analysis is used for the oscillation analysis, and we also use the covariance method. The

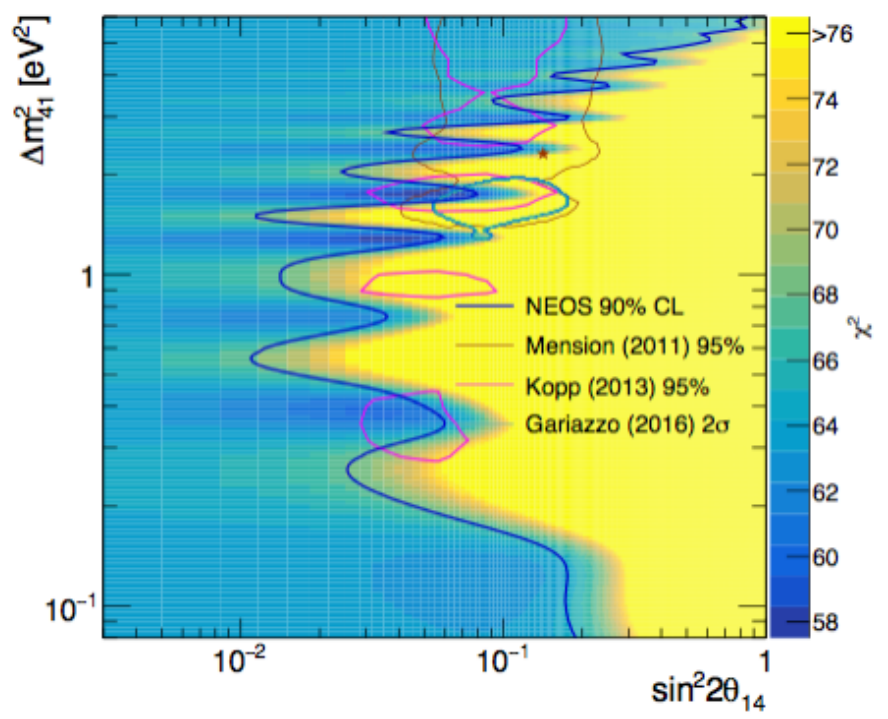

Figure 6: Exclusion limit curve at $90 \% \mathrm{CL}$. with some results of global analysis for sterile neutrino. 
covariance matrix includes statistical uncertainties of reactor-on and -off data, overall normalization for shape-only analysis, background systematic uncetainty, and systematics of energy scale and flux model. The left figure of Figs. [5 shows the statistical and systematic uncertainties with best fit value of oscillation parameters and best fit of RAA. The $\chi_{3 v}^{2}$ with null hypothesis, $\mathrm{H}_{3 v}$, is 64.0 with the number of degrees of freedom (NDF) of 61 , and the $\chi_{\min }^{2} / N D F$ with alternative hypothesis, $\mathrm{H}_{4 v}$, is $57.5 /(61-2)$.

The $\Delta \chi^{2}=\chi_{3 v}^{2}-\chi_{4 v}^{2}$ is 6.5 with two oscillation parameters, and the significance test of the $\Delta \chi^{2}$ is performed with pseudo-experiments because there is correlation between parameters. And then, the right figure of Figs. [ 5 shows that there is no strong evidence of light sterile neutrino in $3+1$ hypothesis with $22 \% p$-value. Finally, we obtain the exclusion limits via raster scan with $\chi^{2}$ distribution as shown in Fig.6.

\section{References}

[1] C. Athanassopoulos et al. [LSND Collaboration], Evidence for $\bar{v}_{\mu} \rightarrow \bar{v}_{e}$ Oscillation from the LSND Experiment at the Los Alamos Meson Physics Facility Phys. Rev. Lett. 70, 3082 (1996).

[2] A. A. Aguilar-Arevalo et al. [MiniBooNE Collaboration], Improved Search for $\bar{v}_{\mu} \rightarrow \bar{v}_{e}$ Oscillations in the MiniBooNE experiment Phys. Rev. Lett. 110, 161801 (2013).

[3] W. Hampel et al. [GALLEX Collaboration], Final Results of the ${ }^{51}$ Cr Neutrino Source Experiments in GALLEX Phys. Lett. B 420, 114-126 (1999).

[4] F. Kaether et al., Reanalysis of the GALLEX Solar Neutrino Flux and Source Experiment Phys. Lett. B 685, 47-54 (2010).

[5] J. N. Abdurashitov et al. [SAGE Collaboration], Measurement of the Response of a Gallium Metal Solar Neutrino Experiment to Neutrinos from a ${ }^{51}$ Cr Source Phys. Rev. C 59, 2246 (1999).

[6] J. N. Abdurashitov et al. [SAGE Collaboration], Measurement of the Response of a Ga Solar Neutrino Experiment to Neutrinos from a ${ }^{37}$ Ar Source Phys. Rev. C 73, 045805 (2006).

[7] G. Mension et al., Reactor Antineutrino Anomaly Phys. Rev. D 83, 073006 (2011).

[8] P. Huber, Determination of Antineutrino Spectra from Nuclear Reactors Phys. Rev. C 84, 024617 (2011).

[8] Th. A. Mueller et al., Improved Predictions of Reactor Antineutrino Spectra Phys. Rev. C 83, 054615 (2011).

[9] J. F. Become and P. Vogel, Angular Distribution of Neutrino Inverse Beta Decay Phys. Rev. D 60, 053003 (1999).

[10] V. I. Kopeikin et al., Components of Antineutrino Emission in Nuclear Reactor Phys. Atom. Nucl. 67, 1963 (2004).

[11] B. Pontecorvo, Inverse Beta Processes and Nonconservation of Lepton Charge JETP 34, 247 (1957).

[12] F. P. An et al. [Daya Bay Collaboration], Measurement of the Reactor Antineutrino Flux and Spectrum at Daya Bay Phys. Rev. Lett. 116, 061801 (2016). 